\title{
Ancora un tentativo di controllo di un periodo sismico (Terremoti della Tolfa del Luglio 1969)
}

\author{
(About a further attempt to control a seismic period - Earthyuakes \\ of the Tolfa Mountains during July 1969)
}

I. MARCFLLI - P. E. VALLE

Ricevuto il 20 Febbraio 1970

Sumary. - The results of an attempt to control the seismic period of the Tolfa Mountains, started on July 2nd 1969, are herein slown.

The attempt was made on the hasis of the intrinsic method and of the elastic rebound theory.

Riassunto. - Vengono esposti i risultati di un tentativo di porre sotto controllo il periodo sismico della Tolfa, iniziato il 2 Luglio 1969.

Il tentativo è stato effettuato sulla base del metodo intrinseco o della teoria dell'" elastic rebound".

\section{INTRODUZIONE.}

In questi ultimi anni in alcmi Paesi sono state intensificate le ricerche connesse alla previsione dei fenomeni sismici.

Non meno importante del problema della previsione è tuttavia il problema del controllo.

Qualche soluzione del problema della previsione potrebbe contenere anche la soluzione del problema del controllo, ma, almeno per ora, le due questioni possono essere affrontate indipendentemente l'una dall'altra.

Una volta che, previsto o meno, un periodo sismico sia iniziato, formulare il problema del controllo significa formulare sostanzialmente le seguenti domande:

1) che cosa è avvenuto?

2) che cosa avviene?

3) che cosa si puod dire su ciò che ancora potrà avvenire? 
Durante il recente periodo sismico siciliano si ̀̀ già cercato di rispondere a queste domande mediante l'impiego del metodo intrinseco e la teoria dell'" elastic rebound ".

I risultati sono stati disoussi in una nota precedente (1), alla quale rimandiamo per tut to quanto qui riteniamo superfluo ripetere.

In tale lavoro è stato anche esposto il concetto di "metodo intrinseco " e la teoria dell' elastic rebound ", sulla base del modello di II. Beniofi.

E inutile qui insistere sulla nota teoria dell' elastic rebound", ma è forse opportuno ricordare che il "metodo intrinseco" si basa sostanzialmente sul fatto che un fenomeno naturale puo essere studiato in sé, al di fuori del tempo convenzionale, assumendo come variabile indipendente il "tempo proprio ", definito operativamente come il numero d'ordine degli eventi in cui il fenomeno si realizza.

Allo scopo di contribuire alla formazione di una documentazione sui tentativi di controllo, in questa nota si espongono i risultati ottenuti applicando al periodo sismico dei Monti della Tolfa, iniziato il 2 luglio 1969, gli stessi metodi utilizzati per il controllo del periodo sismico siciliano, iniziato il 14-I-1968 (*). Sono state qui peraltro apportate alcume semplificazioni, come ad esempio la soppressione della scala delle magnitudo nel diagramma del rendimento attuale; risulta inoltre più chiaramente espresso, nella relativa didascalia, il significato del diagramma di Fig. 5 rispetto all'analogo diagramma di Fig. 11 della nota citata $\left(^{1}\right)$

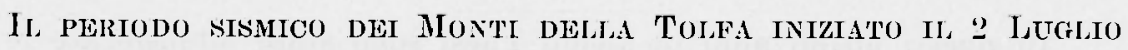
1969.

Ito studio macrosismico di questo breve periodo sismico è stato eseguito da M. De Panfilis $\left({ }^{2}\right)$, il quale lad determinato, tra l'altro, la posizione della regione epicentrale, che viene indicata in Figr. 1, e la profondità ipocentrale, che è risultata di circa $8 \mathrm{~km}$.

11 periodo sismico non ha avuto scosse premonitorie. Si è realizzato con una scossa principale ed una serie di repliche.

Ie Magnitudo delle scosse e il tempo d'arrivo all'Osservatorio di Roma - o all'Osservatorio di Monte Porzio - sono elencate nella Tabella I.

$\left({ }^{*}\right)$ Nella citata nota $\left(^{1}\right)$ vi sono alcuni errori di trascrizione, che però possono essere rilevati abbastanza facilmente. 
Vi è da osservare che in questa Tabella non compare una scossa che si sarebbe verificata il giorno 5 Luglio 1969 , alle ore 04 cirea, perché questa scossa, sebbene segnalata dagli abitanti del loogo, non è stata registrata né dagli strumenti di Roma, né dagli strumenti più sensibili

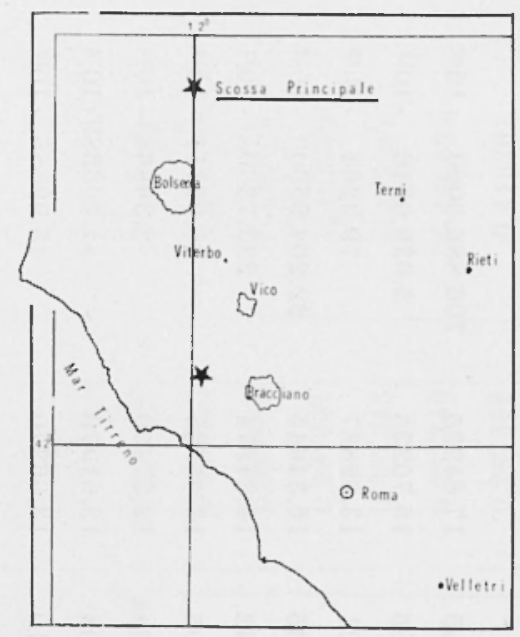

Fig. 1 - Epicentro della scossa principale.

dell'Osservatorio di Monte Porzio, sito a circa $20 \mathrm{~km}$ a Sud di Roma. Tnoltre la scossa avente il numero d'ordine $K_{o}=8$ è incerta.

TL NUMERO D'ORDINE DELIAE SCOSSE IN FUNZZIONE DEL TEMPO.

Mentre le scosse si susseguivano veniva costruito il diagramma di Fig. 2, riportando in ordinate e scala lineare il numero d'ordine o " tempo proprio " delle scosse, e in ascisse e scala logaritmica il relativo tempo origine convenzionale. Le grandezze in questione sono state contate a partire dalla prima scossa.

E da rilevare che nel caso che un periodo sismico inizi con le scosse premonitorie, la scossa principale è facilmente individuabile perchè l'andamento delle repliche nel diagramma in questione ̀̀ assai diverso dall'andamento delle scosse premonitorie, come risulta p. es. dalle Figg. 5 e 6 del lavoro sulla Sicilia (1).

Per i terremoti della Tolfa il grafico costruito faceva peraltro sospettare, sin dalle prime scosse, che il periodo sismico fosse iniziato direttamente con la scossa principale, come poi eflettivamente risultò. 
Tabella I

\begin{tabular}{|c|c|c|c|c|c|c|c|}
\hline $\begin{array}{l}\text { Data } \\
1969\end{array}$ & $\begin{array}{c}K_{o} \\
\text { (No delle } \\
\text { scosse) }\end{array}$ & $\begin{array}{c}K \\
(\text { No delle } \\
\text { repliche })\end{array}$ & $\begin{array}{l}\text { Tempi d'arrivo (*) del- } \\
\text { le prime onde a Roma } \\
\text { (o a M. Porzio) }\end{array}$ &.$I$ & $\begin{array}{c}\log _{10} E= \\
9.154+2.147 \mathrm{I}\end{array}$ & $E$ & $\begin{array}{c}t \\
\text { tempo in mi } \\
\text { nuti dalla } \\
\text { primascossa }\end{array}$ \\
\hline 2 Juglio & 1 & $\begin{array}{c}\text { Scossa } \\
\text { principale }\end{array}$ & $\begin{array}{cccc}\text { h } & \text { m } & \text { s } & \\
09 & 55 & 53 & \text { (Roma) }\end{array}$ & 4,31 & 18,40757 & $2.556 .052,0000 \quad \cdot 10^{12}$ & - \\
\hline ” & 2 & 1 & 100307 (Roma) & 4,05 & 17,84935 & $706.886,8000 \quad \cdot 10^{12}$ & 7,2 \\
\hline$n$ & 3 & 2 & 100814 (Roma) & 3,05 & 15,70235 & $5.039,0640 \cdot 10^{12}$ & 12,3 \\
\hline$n$ & 4 & 3 & 101228 (Roma) & 2,21 & 13,89887 & $79,2264 \cdot 10^{12}$ & 16,6 \\
\hline$n$ & 5 & 4 & $1021(30)$ (Roma) & 3,35 & 16,34645 & $22.204,9500 \quad \cdot 10^{12}$ & 25,6 \\
\hline$"$ & 6 & 5 & 103713 (Roma) & 2,42 & 14,34974 & $223,7380 \cdot 10^{12}$ & 41,3 \\
\hline$n$ & 7 & 6 & 114515 (Roma) & 2,05 & । 3,55535 & $35,92111 \cdot 10^{12}$ & 109,3 \\
\hline$n$ & 8 & 7 & 122700 (M. Porzio) & $1,50 \mathbf{a}$ & 12,37450 & $2,368644 \cdot 101)^{12}$ & 151,1 \\
\hline ” & 9 & 8 & 130145 (Roma) & 2,08 & 13,61976 & $41,166389 \cdot 10^{12}$ & 185,8 \\
\hline 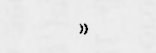 & 10 & 9 & 154433 (Roma) & 2,10 & 13,66270 & $45,99585 \cdot 10^{12}$ & 348,7 \\
\hline$"$ & 11 & 10 & 235613 (M. Porzio) & 2,00 & 13,44800 & $28,05433 \cdot 10)^{12}$ & 840,3 \\
\hline 3 Juglio & 12 & 11 & 061303 (Roma) & 2,00 & 13,44800 & $28,05433 \cdot 10^{12}$ & 1217,2 \\
\hline 9 Jılıglio & 13 & 12 & 173800 (Roma) & 1,50 & 12,37450 & $2,3686+4 \cdot 10^{12}$ & $105+2,1$ \\
\hline$n$ & 14 & 13 & 225017 (Roma) & 1,50 & 12,37450 & $2,368644 \cdot 10)^{12}$ & 10854,4 \\
\hline
\end{tabular}

$\left(^{*}\right)$ I tempi qui indicati si riferiscono all'ora legale adottata in Italia dal 1 Gingno al 27 sett. 1969. 1 tempi corrispondenti all'ora di Greenwich si hamno togliendo 2 ore ai tempi indicati. 
Tabella II

Terremoti della Tolfa - REPLICHE.

\begin{tabular}{|c|c|c|c|c|c|c|}
\hline \begin{tabular}{c}
\multicolumn{1}{c}{$\boldsymbol{K}$} \\
Numero \\
d'ordine \\
delle \\
repliche
\end{tabular} & $\begin{array}{l}\text { (Energia } \\
\text { normalizzata) } \\
\quad x_{k}=\frac{E_{k}}{E_{o}}\end{array}$ & $x_{k}^{1 / 2}$ & $\begin{array}{l}\text { (Deformazione } \\
\text { normalizzata) } \\
\sum_{1}^{k} x_{j}^{1 / 2}=b_{k}\end{array}$ & $\sum_{1}^{k} x_{j}=a_{k}$ & $\eta_{k}=\frac{a_{k}}{b_{k}}$ & $\begin{array}{c}\text { (osservato) } \\
A \eta_{k+1, k}\end{array}$ \\
\hline 1 & 0,2765541546 & 0,525884 & 0,525884 & 0,276554 & 0,525884 & - \\
\hline 2 & 0,0019714246 & 0,044401 & 0,570285 & 0,278526 & 0,488398 & $-0,037486$ \\
\hline 3 & 0,0000309956 & 0,005567 & 0,575852 & 0,278557 & 0,483730 & $-0,004668$ \\
\hline 4 & 0,0086872058 & 0,093205 & 0,669057 & 0,287244 & 0,429327 & $--0,054403$ \\
\hline 5 & 0,0000875326 & 0,009356 & $0,678+13$ & 0,287331 & 0,423534 & $-0,005792$ \\
\hline 6 & 0,0000140533 & 0,003749 & 0,682162 & 0,287345 & 0,421227 & $-0,002307$ \\
\hline 7 & 0,0000009266 & 0,000963 & 0,683125 & 0,287346 & 0,420635 & $-0,000592$ \\
\hline 8 & 0,0000161054 & 0,004013 & 0,687138 & 0,287362 & 0,418201 & $-0,002433$ \\
\hline 9 & 0,0000179948 & 0,004242 & 0,691380 & 0,287380 & 0,415661 & $-0,002540$ \\
\hline 10 & 0,0000109756 & 0,003313 & 0,694693 & 0,287391 & 0,413695 & $-0,001966$ \\
\hline 11 & 0,0000109756 & 0,003313 & 0,698006 & 0,287402 & 0,411747 & 0,001948 \\
\hline 12 & 0,0000009266 & 0,000963 & 0,698969 & 0,287403 & 0,411181 & $-0,000565 \bar{\tau}$ \\
\hline 13 & 0,0000009266 & 0,000963 & 0,699932 & 0,287404 & 0,410617 & $-0,0005642$ \\
\hline
\end{tabular}


Tabella III

Terremoti della Tolfa - REPLICIIE. (Dati osservati e previsii)

\begin{tabular}{|c|c|c|c|c|c|c|c|c|c|c|}
\hline 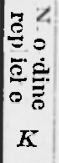 & $\begin{array}{c}x_{0, k+1}^{1 / 2} \\
\text { (previsto) }\end{array}$ & $\begin{array}{c}x_{k}^{1 / 2} \\
\text { (osservato) }\end{array}$ & $\begin{array}{l}\sum r_{f}^{1 / 2}=b_{k} \\
\text { (osservato) }\end{array}$ & $\begin{array}{c}\Sigma x=a_{i} \\
\text { (osservato) }\end{array}$ & $\begin{array}{c}x_{m}^{1 / 2} \\
\text { (previsto) }\end{array}$ & $\begin{array}{c}.1 \eta_{m} \\
\text { (previsto) }\end{array}$ & $\begin{array}{c}\text { In } \\
\text { (osservato) }\end{array}$ & $\begin{array}{c}.1 \eta_{M} \\
\text { (previsto) }\end{array}$ & $\begin{array}{c}r_{m} \\
(\text { previsto) }\end{array}$ & $\begin{array}{c}r \\
\text { (osservato) }\end{array}$ \\
\hline 1 & - & 0,525884 & 0,525884 & 0,276554 & - & - & - & - & - & - \\
\hline 2 & 0,5259 & 0,044401 & 0,570285 & 0,278526 & 0,2178 & $-0,09023$ & $-0,03749$ & 0,3107 & $-0,2904$ & $-0,1206$ \\
\hline 3 & 0,4884 & 0,005567 & 0,575852 & 0,278557 & 0,2067 & -0.07494 & $-0,004668$ & 0,3258 & -0.2300 & $-0,0143$ \\
\hline 4 & 0,4837 & 0,093205 & 0,669057 & 0,287244 & 0,2053 & $-0,07318$ & $-0,05440$ & 0,3276 & $-0,2234$ & $-0,1661$ \\
\hline$\overline{5}$ & 0,4293 & 0,009356 & 0,678413 & 0,287331 & 0,1882 & $-0,05294$ & $-0,005792$ & 0,3419 & $-0,1548$ & $-0,0169$ \\
\hline 6 & 0,4235 & 0,003749 & $0,682 \perp 62$ & 0,287345 & 0,1862 & $-0,05111$ & $-0,002307$ & 0.3435 & -0.1488 & $-0,0067$ \\
\hline 7 & 0,4212 & 0,000963 & 0,683125 & 0,287346 & 0,1854 & $-0,05040$ & $-0,0005922$ & $0,34+1$ & $-0,1465$ & $-0,0017$ \\
\hline 8 & 0,4206 & 0,004013 & 0,687138 & 0,287362 & 0,1852 & $-0,05021$ & $-0,002433$ & 0,3442 & $-0,1+59$ & $-0,0071$ \\
\hline 9 & $0,+182$ & 0,004242 & 0,691380 & 0,287380 & 0,1844 & $-0,04947$ & $-0,002 \tilde{5} 40$ & 0,3448 & $-0,1435$ & $-0,0074$ \\
\hline 10 & 0,4157 & 0,003313 & 0,694693 & 0,287391 & 0,1835 & $-0,04869$ & $-0,001966$ & 0,3455 & $-0,1409$ & $-0,0057$ \\
\hline 11 & 0,4137 & 0,003313 & 0,698006 & 0,287402 & 0,1828 & $-0,04810$ & $-0,001948$ & 0,3460 & $-0,1390$ & $-0,0056$ \\
\hline 12 & 0,4117 & 0,000963 & 0,698969 & 0,287403 & 0,1821 & $-0,04752$ & $-0,0005657$ & 0,3464 & $-0,1372$ & $-0,0016$ \\
\hline 13 & 0,4112 & 0,000963 & 0,699932 & 0,287404 & 0,1819 & $-0,04735$ & $-0,0005642$ & 0,3466 & $-0,1366$ & $-0,0016$ \\
\hline 14 & $(0,4106)$ & - & - & 一 & $(0,1817)$ & $(-0,04718)$ & - & $(0,3467)$ & $(-0,1361)$ & - - \\
\hline
\end{tabular}


LA DEFORMAZIONE LIBERATA DURANTE IL PROCESSO DELLE REPLICIF.

Isa deformazione liberata durante il processo delle repliche è statar considerata soltanto dal punto di vista intrinseco e ció in funzione del numero d'ordine $K$ delle repliche, contato a partire dalla prima replica.

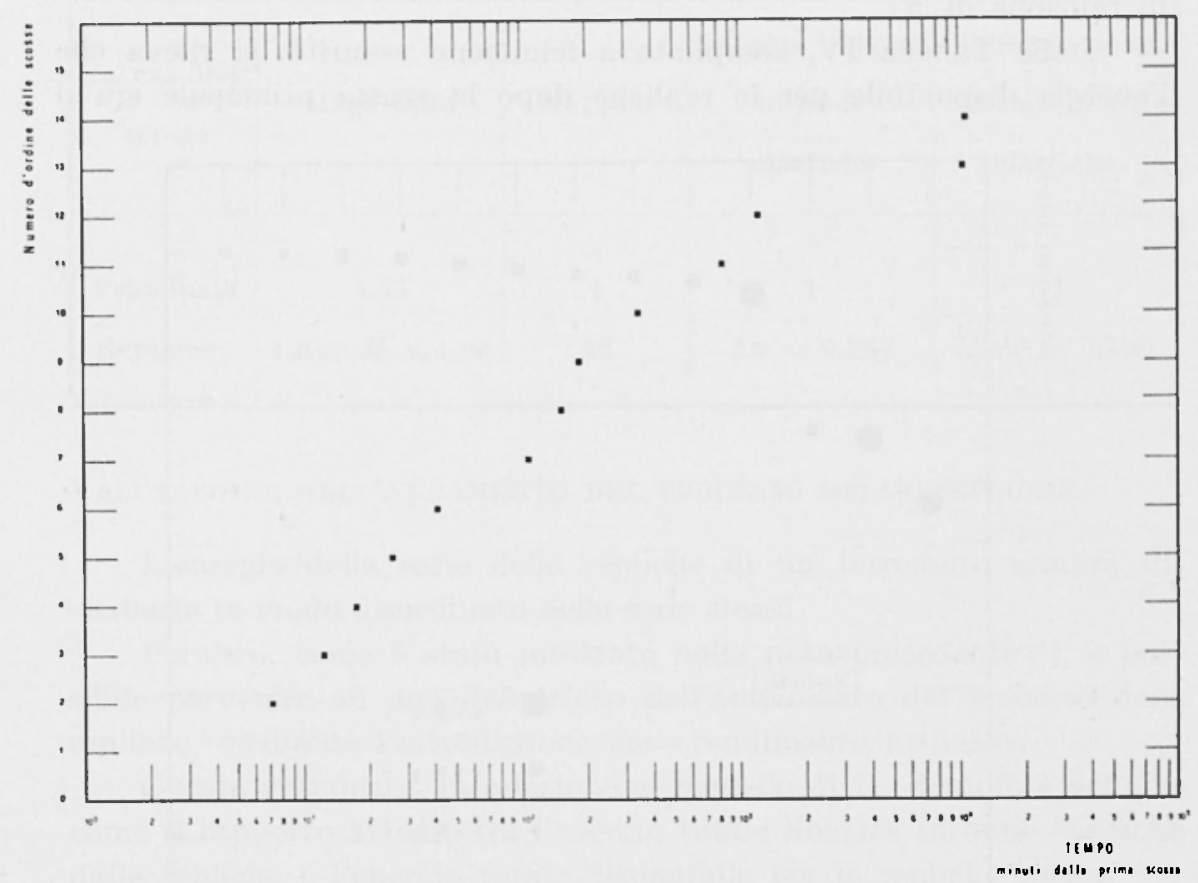

Fig. 2 - Numero d'ordine delle scosse in funzione del tempo.

Le energie sono state calcolate mediante la relazione

$$
\log E=a M+b
$$

con $a=2,147$ e $b=9,154\left(^{3}\right)$.

Detta $E_{o}$ l'energia liberata dalla scossa principale, e $E_{\text {J l'energia }}$ liberata da una generica replica, introdotto il rapporto

$$
x_{j}=\frac{E_{j}}{E_{o}}
$$


che rappresenta l'energia normalizzata della j.ma replica, è stato costruito il diagramma di Fig. 3, nel quale è riportata la deformazione normalizzat:

$$
S_{k}=\sum_{1}^{k} x_{j}^{1 / 2}
$$

in funzione di $K$.

Dalla Tabella $I V$, compilata a fenomeno esaurito, si rileva che l'energia disponibile per le repliche dopo la scossa principale era il

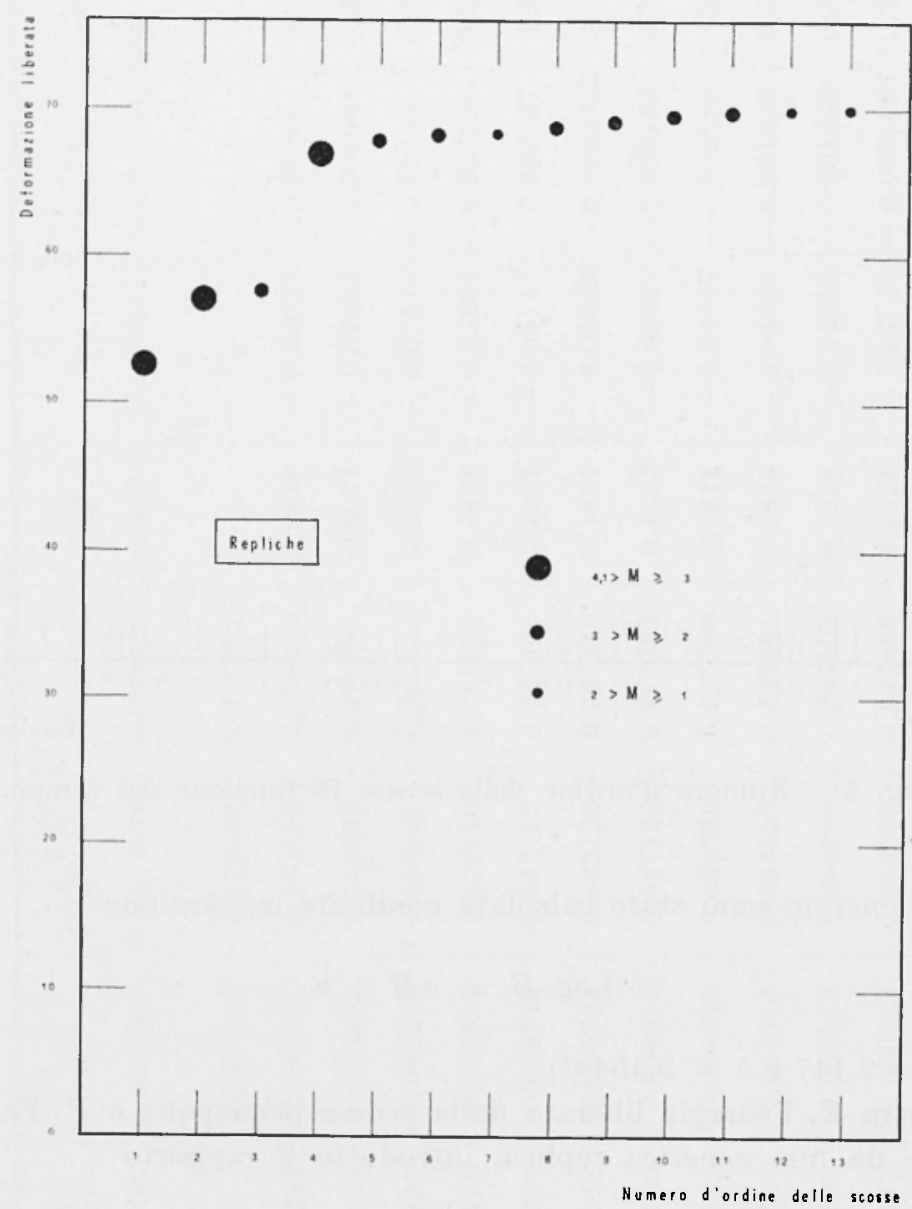

Fig. 3 -- Percentuale della deformazione liberata durante il processo delle repliche, in funzione del loro numero d'ordine. 
$70 \%$ della energia liberata dalla scossa principale stessa. Di questa energia soltanto il $41 \%$ è stata liberata in onde elastiche dalle repliche, il rimanente $59 \%$ si è trasformata in calore.

Dall'andamento del grafico di Fig. 3 si può ritenere che la deformazione liberata dalle repliche era di pressione (trazione) $\left(^{4}\right)$.

Tabella IV

\begin{tabular}{|c|c|c|c|c|}
\hline $\begin{array}{c}\text { Natura delle } \\
\text { seosse }\end{array}$ & Magnitudo & Numero & $\begin{array}{c}\text { Energia liberata } \\
\text { in onde } \\
\text { elastiche }\end{array}$ & $\begin{array}{c}\text { Deformazione } \\
\text { liberata in onde } \\
\text { elastiche }\end{array}$ \\
\hline $\begin{array}{c}\text { Principale } \\
\text { Repliche }\end{array}$ & $1,5 \leqslant M \leqslant 4,05$ & 13 & $\Sigma x=0,287$ & $\Sigma x^{1 / 2}=0,700$ \\
\hline
\end{tabular}

VALUTAZIONE DELL'ANDAMENTO DEL PROCESSO DELLE REPLICIE.

L'energia della serie delle repliche di un terremoto sembra distribuita in modo disordinato nella serie stessa.

Peraltro, come è stato mostrato nella nota precedente ( $\left.{ }^{1}\right)$, ̀̀ possibile pervenire ad una definizione dell'andamento del processo delle repliche, mediante l'introduzione del "rendimento attuale ".

Questa grandezza, in relazione al modello di $\mathrm{H}$. Benioff, è definita come il rapporto attuale tra l'energia totale liberata in onde elastiche dalle repliche e l'energia totale disponibile per le repliche stesse.

Se si introducono le energie normalizzate delle repliche

$$
x_{j}=\frac{E_{j}}{E_{o}}
$$

dove $E$, ̀̀ l'energia di una generica replica ed $E_{o}$ la somma dell'energia della scossa principale e delle eventuali scosse premonitorie, il rendimento attuale " $\eta_{k}$ " ̀̀ dato dalla

$$
\eta_{k}=\frac{\frac{\stackrel{k}{j}_{j} x_{j}}{\sum_{i} x_{j}^{\frac{1}{2}}}}{\sum_{1}}
$$


Posto per' sempliciti

$$
\frac{v_{i}}{1} x_{j}=a_{k}, \quad \sum_{1}^{k} \sum_{j} e_{j}=b_{k}
$$

$\left(b_{k}=S_{k}\right)$

si puo anche scrivere

$$
\eta_{k}=\frac{a_{k}}{b_{k}} .
$$

Si supponga verificata la $k^{m a}$ replica e di aver calcolato il rapporto $\eta_{k}$. Dopo l'eventuale $(k+1)^{\text {ma }}$ replica tale rapporto assumerà il valore

$$
\eta_{k+1}=\frac{a_{k}+x_{k+1}}{b_{k}+x_{k+1}^{1}}
$$

Il rapporto in questione varierà pertanto di

$$
\Delta \eta_{k-1, k}=\frac{x_{k+1}^{\frac{1}{2}}\left(x_{k+1}^{\frac{1}{2}}-\eta_{k}\right)}{b_{k}+x_{k+1}^{-\frac{1}{2}}}
$$

Si vede subito che l'andamento del rendimento attuale in funzione del numero d'ordine delle repliche consente di definire il processo delle repliche stesse come rescente, stazionario o decrescente a seconda che risulti rispettivamente

$$
\begin{aligned}
& \Delta \eta_{k+1, k}>0 \\
& \Delta \eta_{k+1, k}=0 \\
& \Delta \eta_{k+1, k}<0 .
\end{aligned}
$$

Ossia, indicando con

$$
x_{o, \bar{k}+i}^{\frac{1}{2}}=\eta_{k}
$$

la deformazione the dovià essere liberata dalla eventuale $(k+1)^{m a}$ replica affinché il processo risulti stazionario, si avrà

$$
x_{k+1}^{1}>x_{0, k+1}^{1}, \quad x_{k+1}^{1}=x_{0, k+1}^{1}, \quad x_{k+1}^{1}<x_{0, k+1}^{1}
$$


a seconkla che il fenomeno si presenti, in questa successiva replican, rispettivamente rrescente, stazionario oppure decrescente.

Poiché $x_{k+1}^{1}$ rapleresenta la deformazione (normalizzata) liberata nella eventua le $(k+1)^{\text {ma }}$ replica, il suo valore si cablcola immediatamente, e questo valore, posto a confronto con il $x_{o, k+1}^{1}=\eta_{k}$ grià calcolato fino alla $k^{m a}$ replicar dice subito in quale delle tre fasi possibili viene a trovarsi il processo delle repliche stesse.

Se quindi nel corso del verificarsi delle repliche si costruisce un grafico riportando in ascisse il loro numero d'ordine e in ordinate il corrispondente valore del rendimento attuale e se a questo si sovrap)pone il grafico delle deformazioni (normalizzate) si ̀̀ in grado di controllare, all'insorgere di ogni repling, se il processo delle repliche stesse si trovi in una fase crescente, o stazionaria, oppure decrescente.

Nel caso dei terrenoti della Tolfa il processo delle repliche a stato sempre decrescente, come risulta dalla Fig. $t$.

PRENISIONE DELL'TNTERTALO IN CUI POTRA ESSTRE COMPREA LA

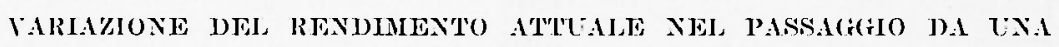
REPLICA ALLA EVENTUALE SUCCESSIVA.

Nella citata nota sul periodo sismico siciliano si è anche rilevato che la valiazione del rendimento attuale da una replica bia alla eventuale successiva $(k+1)^{\text {ma }}$

$$
\Delta \eta_{k+1, k}=\frac{x_{k+1}^{1}\left(x_{k+1}^{\frac{1}{2}}-\eta_{k}\right)}{b_{k}+x_{k+1}^{\frac{1}{2}}}
$$

¿ una funzione continua della variabile $x_{k+1}$ nel suo intervallo di definizione $0 \vdash-1$, la quale ha un minimo

$$
\Delta \eta_{m}=-\frac{x_{m}}{b_{k}}
$$

che assume per

$$
x_{k+1}=x_{m}
$$

$(011$

$$
x_{m}=b_{k}^{2}\left(-1+\sqrt{1+\frac{\eta_{k}}{b_{k}}}\right)^{2} .
$$




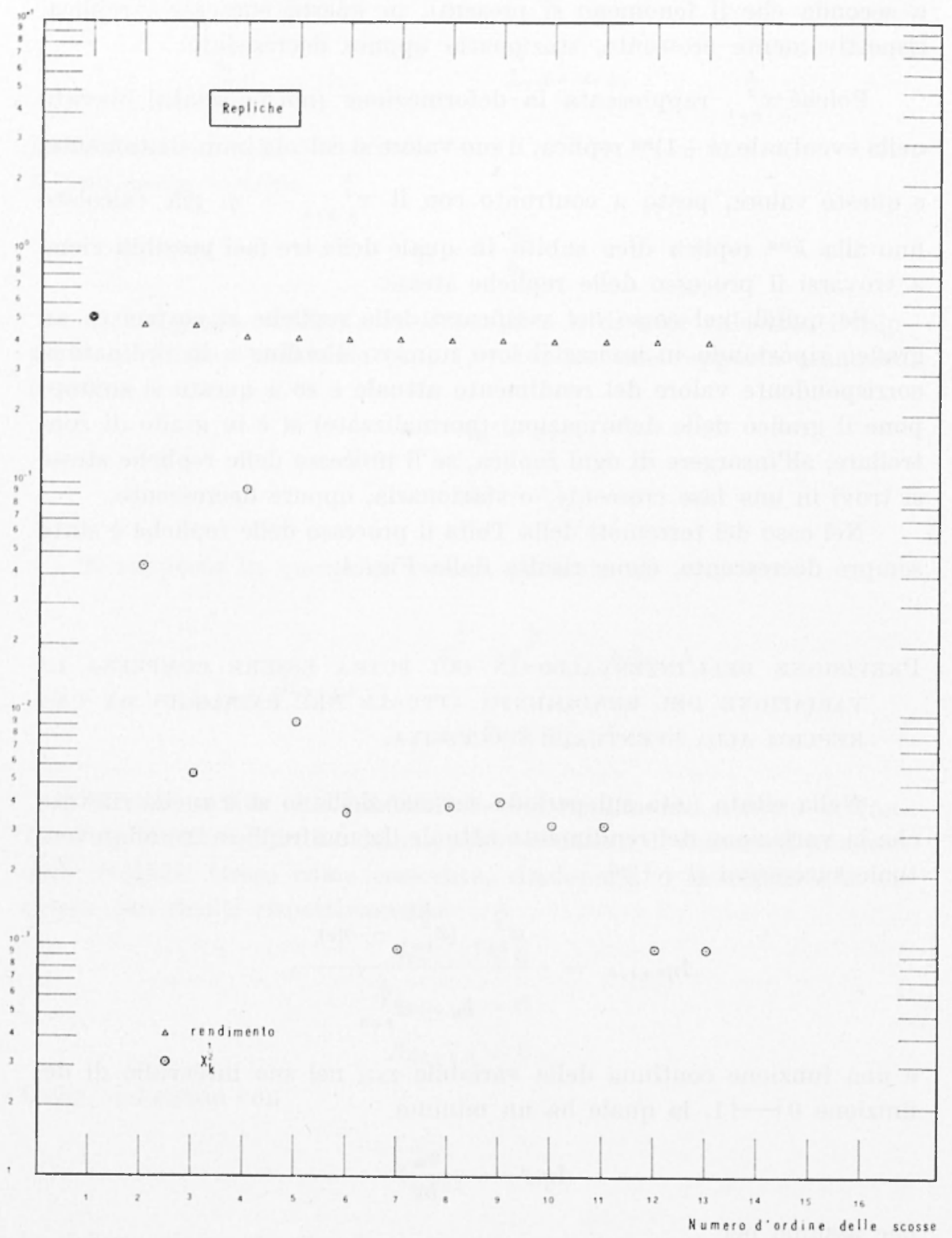

Fig. 4 - Rendimento attuale del processo delle repliche, in funzione del loro numero d'ordine. 
Passa per lo zero quando

$$
x_{k+1}-x_{0, k+1}=\eta_{k}^{2}
$$

e poi cresce fino a raggiungere il suo valore piu grande

$$
\Delta \eta_{M}=\frac{1-\eta_{k}}{1+b_{k}}
$$

che assume per

$$
x_{k+1}=1
$$

Pertanto lintervallo entro il quale può variare il rendimento attuale nel passaggrio del processo da ma replica alla eventule successiva puó essere previsto in quanto vale sempre la limitazione

$$
\Delta \eta_{m} \leqslant \Delta \eta_{k+1, k} \leqslant \Delta \eta_{M}
$$

Se si introduce la "variazione ridotta del rendimento attuale $\Delta \eta / 1 \eta_{M} "$ il cui valore minimo prevedibile è definito dalla

$$
\ddot{r}_{m}=\frac{\Delta \eta_{m}}{\Delta \eta_{M}}
$$

da confrontare con l'eventuale analoga variazione ridotta osservatu

$$
r=\frac{\Delta \eta_{k+1, k}}{\Delta \eta_{M}}
$$

si può prevedere l'intervallo entro il quale potrà essere compresa la grandezza $r$ nel passaggio del processo delle repliche dalla lima alla $(k+1)^{m a}$, perché sussiste la limitazione

$$
r_{m} \leqslant r \leqslant 1 .
$$

Evidentemente per

$$
r_{m} \leqslant r<0
$$

il processo è decrescente.

Se

$$
r=0
$$

il processo è stazionario e se

$$
0<r \leqslant 1
$$

il processo ì crescente. 
Landamento di $r_{m}$ in funzione del numero dordine delle repliche puo fornire qualche indicazione sulla possibilità che il processo cambi fase.

Se fino arl un certo valore di $k$ il processo delle repliche è risultato decrescente $\left(r_{m} \leqslant r<0\right)$, ma $r_{m}$ i crescinto mpirlamente avvicinandosi al valore zero, sussiste il pericolo rhe il processo stesso cambi fase, passando da decrescente a rescente. E possibile peraltro che il proresso cambi fase anche se non si presentano le suddette condizioni.

Nel caso del periodo sismieo dei Monti della Tolfa il processo è stato sempre decrescente, come is stato già rilevato. Il parametro $r_{m}$ ¿ resciuto piuttosto lentamente, come mostra la Fig. 5, e ciò ha

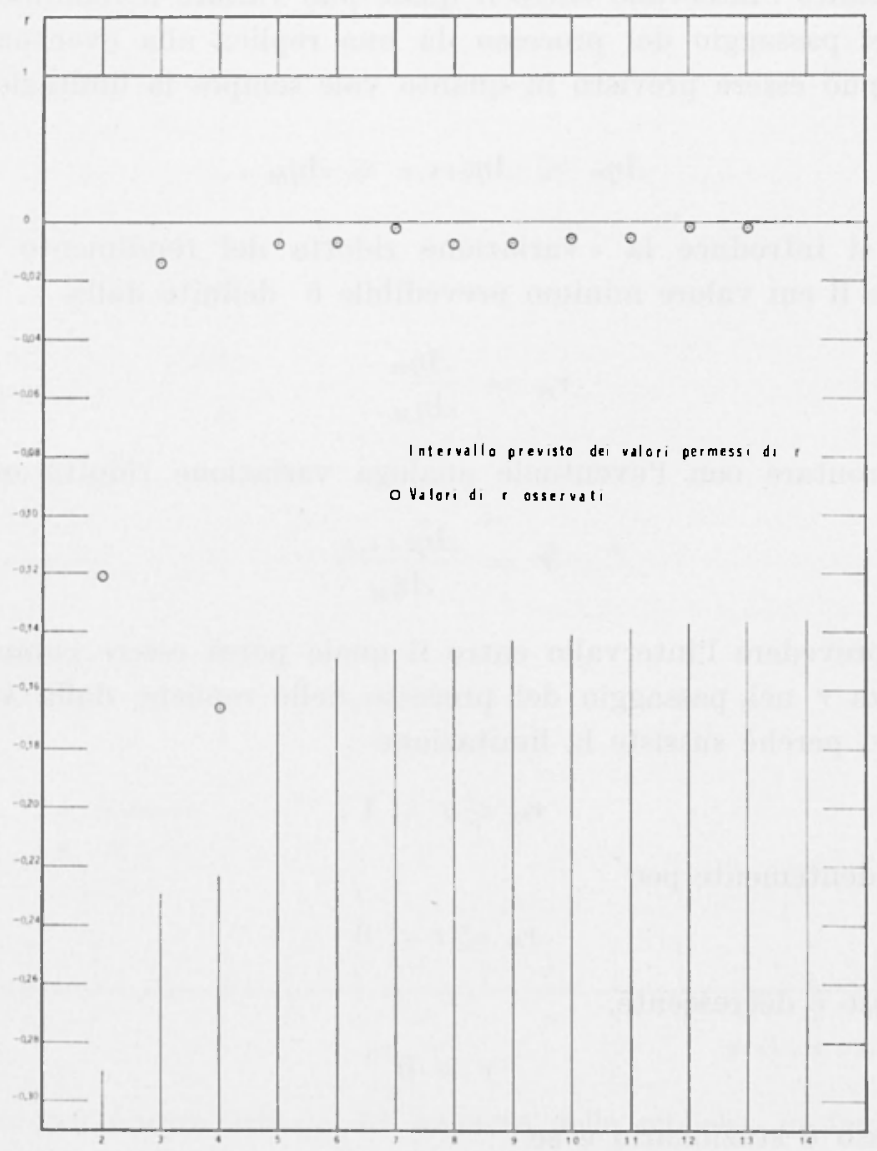

Numero d'ordine delle scosse

Fig. 5 - Intervallo previsto dei valori permessi del parametro $r$ e valori osservati, in funzione del numero d'ordine delle repliche. 
consentito di ritenere, dopo ogni replica, che difficilmente il processo avrebbe cambiato fase.

\section{Osservizioni.}

Il periodo sismico dei MLonti della Tolfa iniziò alle 09":55m (ore legali) del giorno 2 Juglio 1969. Alle ore 1 th circa era gia stato costruito parte del grafico di Fig. 2 , con le scosse verificatesi fino a quel momento.

Come si è già accennato, questo grafico indnceva a ritenere clue il periodo era iniziato direttamente con la scossa principale e che il fenomeno el"a già in fase di repliche.

Ia pratica certezza si ebbe dagli alti grafici costruiti il giorno successivo.

Vi è da rilevare che all'inizio di un periodo sismico sussiste sempre il dubbio se le prime scosse siano premonitorie o meno. Difatti l'intervallo di tempo che intercorre tra la prima scossa premonitoria e la scossa principale può essere molto breve, ma può durare anche qualche giorno, come mostrano i poclii dati contenuti nella Tabella V.

Tabella V

\begin{tabular}{|c|c|c|c|c|}
\hline Periodo sismico & $\begin{array}{l}\text { Magnitudo } \\
\text { della scossa } \\
\text { principale }\end{array}$ & $\begin{array}{l}\text { Intervallo di } \\
\text { tempo tra la } \\
\text { prima scossa } \\
\text { premonitoria } \\
\text { e la scossa } \\
\text { principale }\end{array}$ & $\begin{array}{c}\text { Numero } \\
\text { delle scosse } \\
\text { premonitorie }\end{array}$ & $\begin{array}{l}\text { Limite inf. } \\
\text { della } \\
\text { Magnitudo } \\
\text { delle scosse } \\
\text { premonitorie }\end{array}$ \\
\hline & & (ore) & & \\
\hline $\begin{array}{l}\text { Palombara } \\
\text { Sabina }\left(^{5}\right)(1901)\end{array}$ & 6 & 70 & $\tau$ & 2,4 \\
\hline $\begin{array}{l}\text { Termini } \\
\text { Imerese }\left({ }^{(0)}\right) \\
\qquad(1906)\end{array}$ & 4,8 & 70 & 5 & 2,9 \\
\hline $\begin{array}{c}\text { Irpinia }\left({ }^{7}\right) \\
(1962)\end{array}$ & 6,2 & 2,4 & 3 & 4,5 \\
\hline Sicilia $\left({ }^{1}\right)(1968)$ & 6 & 13 & 7 & 3 \\
\hline Tolfa (1969) & 4,3 & - & 0 & - \\
\hline
\end{tabular}




\section{BIBIIOGRATIA}

(1) Varte P. E., Tentativo di controllo del periodo sismico siciliano iniziato il 1.4 Gennato 1968. "Annali di Geofisica ", XXII, I, (1969).

(2) DE Paxfidrs M., Cn periodo sismico nella zona dei Monli della Tolfa. "Annali di Geofisica", XXII, 3. (1969).

(3) Di Firappo D). - Marcerta L., Magnitudo ed energia dei lerremoti in Ilalia. "Annali di Geofisica ", III, 3, (1950).

(1) BexiofF H., Earthquakes and rock creep. "Bull. Seism. Soc. Amer.", XLI, 31-62, (1951).

(5) Caxcaxi A., Sul periodo sismico iniziatosi il 2.4 Aprile 1901 sul territorio di Palombara Sabina. "Boll. Soc. Sism. Italiana ", VII, 169-193, (1901).

(6) ('Iorano M., Orografia, geologia e tettonica delle zone seosse dai terremoli di Termini Imerese del Setlembre 1906. "Boll. Soe. Sism. Italiana ", XIII, I62-I83, (1908).

(7) D) JiLIPPo D. - PERoxact $\mathrm{F}$., Indagine preliminare della natura fisica del fenomeno che ha originato il periodo sismico Irpino dell'agosto 1962. "Annali di Geofisica" XVI, + (1963). 